\title{
The Study of Experiences during Pregnancy in a Woman with Type 1 Diabetes
}

\author{
Ikuko Nishio ${ }^{*}$, Daisaku Nishimoto', ${ }^{1}$ Masami Chujo² \\ ${ }^{1}$ School of Health Sciences, Faculty of Medicine, Kagoshima University, Kagoshima City, Japan \\ ${ }^{2}$ School of Health Sciences, Faculty of Medicine, Tottori University, Yonago City, Japan \\ Email: *ikuko@halth.nop.kagoshima-u.ac.jp
}

How to cite this paper: Nishio, I., Nishimoto, D. and Chujo, M. (2020) The Study of Experiences during Pregnancy in a Woman with Type 1 Diabetes. Open Journal of Nursing, 10, 1095-1108. https://doi.org/10.4236/ojn.2020.1011078

Received: October 3, 2020

Accepted: November 24, 2020

Published: November 27, 2020

Copyright $\odot 2020$ by author(s) and Scientific Research Publishing Inc. This work is licensed under the Creative Commons Attribution International License (CC BY 4.0).

http://creativecommons.org/licenses/by/4.0/

\begin{abstract}
Background: This study aimed to elucidate the experiences of a woman with type 1 diabetes during the course of her pregnancy. Method: This case study involved 1 participant. Data were collected through a semi-structured interview. Results: The data of experiences during pregnancy of Mrs. A comprised 20 codes, 5 categories, and 11 subcategories. The 5 categories were [abnormalities in the delicate body], [difficulty in blood glucose control for safe childbirth], [the burden of visiting both obstetrics] and [internal medicine departments], and [hope for safe childbirth]. Conclusion: The categories extracted in this study included terms that suggested negative experiences, such as "abnormality", "difficulty", "inconvenience", and "burden". Mrs. A might have used these negative terms due to the difficulties in controlling her blood glucose levels.
\end{abstract}

\section{Keywords}

Type 1 Diabetes, Experiences, Pregnancy, Insulin

\section{Introduction}

In Japan, the annual change in the number of registered type 1 diabetes patients has been gradually increasing since 2001 [1]. Women are more prone to type 1 diabetes than men: the male-to-female ratio of patients with the disease is 1:1.4 1.5 [2]. Type 1 diabetes requires treatment with insulin. It is possible to prevent the onset and progression of diabetic complications by controlling blood glucose levels within normal ranges. Patients with type 1 diabetes need to "live with the disease" through their life course. Living with type 1 diabetes means accepting the disease, incorporating insulin therapy in life, and restructuring life in accordance with treatment [3]. 
Women with type 1 diabetes tend to prioritize housework and child-rearing, worry about others' opinions, and develop a sense of lack because of their roles as a mother, wife, and daughter-in-law [4]. They feel that they cannot manage time freely and that their activities are restricted, as they need to constantly control their blood glucose levels within normal ranges. Due to such limitations in everyday life, women with type 1 diabetes are likely to: discontinue treatment; give up on improving their lifestyle habits; and continue to suffer from poor health with uncontrolled blood glucose levels [5]. These findings suggest that women with type 1 diabetes tend to experience difficult situations in which they have no choice but to engage in behaviors that do not help improve their health. This is because such women prioritize their roles in the family and the community [6].

In recent years, it has become possible for an increasing number of women with type 1 diabetes to have planned pregnancy through strict blood glucose control. Despite this, it is still difficult for women with the disease to become pregnant and give birth. This is because women with the disease who wish to become pregnant are required to practice self-care under medical supervision that involves every aspect of daily living, such as long-term strict blood glucose control prior to pregnancy until delivery, health management during pregnancy, and the prevention of complications. Moreover, studies have found that it is highly likely that women with type 1 diabetes experience unplanned pregnancy [7], and that blood glucose levels in women with the disease are poorly managed prior to pregnancy and during pregnancy [8]. The experience of pregnancy and giving birth allows every woman to feel happy whilst imagining a life with her newborn. However, women with type 1 diabetes are likely to experience psychological burdens because their disease may affect and even worsen the health of the fetus.

While controlling blood glucose levels is crucial for pregnant women with type 1 diabetes, it is assumed that such women are under psychological pressure in relation to the impact of their condition on pregnancy. There is a dearth of research that focuses on narrating the experiences of women with type 1 diabetes during pregnancy. Little is known about such experiences. Hence, this study examines effective nursing support by exploring a case of planned pregnancy in a woman with type 1 diabetes.

\section{Aims}

The aims of this study were to elucidate experiences during pregnancy through the delivery of a woman with type 1 diabetes.

\section{Methods}

\subsection{Design}

This study is a case study of one participant. 


\subsection{Participant and Recruitment}

Potential participants were women with type 1 diabetes who were visiting the department of diabetes medicine of a general hospital on an outpatient basis. The hospital has 666 beds and a day average of 1120 outpatients. Eligible participants were required to experience pregnancy and delivery following the onset of the disease. The head physician of the outpatient unit was requested to select eligible participants. One woman with type 1 diabetes agreed to participate in this study.

\subsection{Case Study}

The participant, Mrs. A, is a housewife in her 20s. She gave birth to her second child, who was at eight months of age at the time of this study. Before having her second child, Mrs. A was living with her husband, their first child, and parents-in-law. Mrs. A married and gave birth to her first child in her early 20s. She was never found to have any abnormalities in health check-ups before the onset of type 1 diabetes, which began in her late 20s. Since Mrs. A wished to have a second child, she started using an insulin pump under her doctor's supervision, and strictly controlled her blood glucose levels. Thereafter, she naturally became pregnant while her blood glucose levels were maintained within normal ranges. As of March 2018, Mrs. A's HbAlc (NGSP) was 6.6\%, and she had no diabetic complications.

\subsection{Data Collection}

Data were collected through a semi-structured interview with Mrs in March, 2018. A. The interview was held in an outpatient waiting room where privacy was maintained. One interview was held, and it lasted for 60 minutes. In the interview, the following questions were asked to Mrs. A: "Can you please tell me about your life from the time when you wished to become pregnant to when you gave birth to your baby?" and "Can you also tell me about your thoughts during those times?" In response to the questions, Mrs. A gave a free narrative. Her narrative was audio-recorded upon her permission. The interview was held in March 2018.

\subsection{Data Analysis}

Data obtained through the semi-structured interview were transcribed and analyzed. The participant's descriptions of her experiences during pregnancy were extracted and coded. Coded segments were then grouped by commonality and relevance, and each group was given a name. Thereafter, subcategories were extracted from the groups. Subsequently, segments in subcategories were grouped by commonality and relevance, and each group was given a name. Thereafter, categories were extracted from the subcategories. Also, storylines were created to indicate the mutual relationship between the subcategories and categories, and provide an overview thereof. In the process of analysis, the researchers checked 
with each other to make sure that the transcript was appropriately interpreted, coded, categorized, and sub-categorized. Additionally, the researchers ensured the reliability and validity of this study by regularly receiving supervision from an expert in nursing care for patients with diabetes. The expert has substantial experience in qualitative research.

\subsection{Ethical Considerations}

Prior to the commencement of this study, the participant received an explanation of the following aspects both in writing and orally: the objectives of the study; the voluntary nature of participation; privacy considerations; and the participant's right to withdraw from the study at any time. Consent for participation was also obtained from the participant. The interview was conducted at a place where the conversation could not be heard by others. The interview was audio-recorded after permission was obtained from the participant. The interview data was securely stored in a lockable room. Also, this study was conducted after having been approved by the ethics review committee of Tottori University beforehand in March, 2018.

\section{Results}

\subsection{Experiences during Pregnancy of a Patient with Type 1 Diabetes}

1) The data of experiences during pregnancy of Mrs. A was composed of 20 codes, 11 subcategories, and 5 categories. In what follows, codes, subcategories, and categories are shown in $<>$, “”, and [ ] respectively. Mrs. A's narrative is shown in italics.

\section{1) Abnormalities in the delicate body}

This category comprised the following two subcategories: "increased sensitivity to pain" and "increased skin vulnerability". The subcategory "increased sensitivity to pain" had one code, i.e. < development of pain characteristic to injection>; "increased skin vulnerability" had two codes, namely, <sensitive skin due to gestational itch $>$ and $<$ remaining scars from repeated injections at the same site>.

\section{$<$ Development of pain characteristic to injection>}

- As I had a large lumpy belly during pregnancy, my belly hurt depending on the (injection) site. I occasionally felt that it was inconvenient. $<$ Sensitive skin due to gestational itch>

- I had pregnancy-related itch during pregnancy. It was a really bad (itch). It is still causing eczema and irritation from time to time.

$<$ Remaining scars from repeated injections at the same site $>$

- I could not use needles at more than one site during pregnancy, which left scars on my skin, and the tone of the skin changed there. I now can use needles at different sites, so they (scars) have faded well.

\section{2) Difficulty in blood glucose control for safe childbirth}


This category was composed of the following four subcategories: "requiring a large increase in insulin doses", "large fluctuations in blood glucose levels", "using different tools in different stages of pregnancy", and "requiring strict blood glucose control during pregnancy". The subcategory "requiring a large increase in insulin doses" had one code, which is <insulin not working>; "large fluctuations in blood glucose levels" had two codes, namely <a surge in blood glucose levels $>$ and $<$ frequent hypoglycemia due to strict tolerance levels $>$. The subcategory "using different tools in different stages of pregnancy" had two codes, namely <insulin therapy limited to pregnancy> and < transition to an insulin pump for safe childbirth $>$. The subcategory "requiring strict blood glucose control during pregnancy" had the following three codes: <setting strict HbAlc goals in order to be allowed to become pregnant>, <setting strict tolerance during pregnancy $>$ and $<$ requiring hospitalization for blood glucose control $>$.

\section{<Insulin not working>}

- I had to increase my insulin dose by three times. Prior to pregnancy, I had been receiving 8 or 7 (units) but had to receive up to 21 to 25 (unites) during pregnancy. My (insulin) dose suddenly increased. Unless I had the dose of insulin increased, my blood glucose levels would not have stabilized.

$<$ A surge in blood glucose levels $>$

- My blood glucose levels went up when I was pregnant. They (blood glucose levels) usually remain at or below 180 after a bolus. However, when I was pregnant, they (blood glucose levels) suddenly went up to 200 and went high after eating. One day, I found my blood glucose levels very high. I thought that I might have become pregnant. So, I used a test kit, which showed a positive result. I was surprised.

<Frequent hypoglycemia due to strict tolerance>

- I started having hypoglycemia as soon as I became pregnant. In my case, it was not like hypoglycemia occurred gradually.

<Insulin therapy limited to pregnancy>

- I self-injected insulin for a period of half a year after I was diagnosed with type 1 diabetes. I received drips when I gave birth to my baby. I self-injected insulin before and after delivery, which I had not used for a while.

$<$ Transition to an insulin pump for safe childbirth>

- In order to strictly control blood glucose levels during pregnancy, I switched the therapy to an insulin pump. I self-injected insulin from the time when I had an onset of the disease through to when I became pregnant. It was from February when I was 27 years old through to November when I was 28 years old.

<Setting strict HbA1c goals in order to be allowed to become pregnant>

- This time, I became pregnant after I developed type 1 diabetes. I became pregnant after my doctor allowed me to do so by setting an HbA1c goal of less than $6 \%$.

$<$ Setting strict tolerance during pregnancy $>$ 
- I controlled my blood glucose levels so that my doctor would allow me to become pregnant. It was hard to control them, but am now happy that I gave birth to a healthy baby.

<Requiring hospitalization for blood glucose control>

- I did not have obvious (hypoglycemic) symptoms during pregnancy. They just became less obvious during that time because I did not feel the symptoms before my blood glucose levels became low. However, I frequently had hypoglycemia.

3) Inconvenience in everyday life due to a large belly

This category comprised one subcategory, which is "inconvenience in everyday life due to a large belly". The subcategory was composed of the following two codes: <choosing the right clothes to hide the insulin pump $>$ and $<$ the large belly was pushing the site where the insulin pump was attached against objects $>$.

<Choosing the right clothes to hide the insulin pump>

- I wore long clothes during pregnancy so that I could hide the insulin pump. $<$ The large belly was pushing the site where the insulin pump was attached against objects>

- I had a large belly during pregnancy. (The attachment part of) the insulin pump hurt my belly while I was cooking when I wore it on the right side of my body. The insulin pump was in my way, so I needed to put it in the pocket of my clothes.

4) The burden of visiting both obstetrics and internal medicine departments

This category comprised one subcategory, namely "visiting both obstetrics and internal medicine departments being daunting". The subcategory was composed of the following two codes: $<$ more frequent doctor visits $>$ and $<$ increased medical bills $>$.

$<$ More frequent doctor visits>

- I had to visit both obstetrics and internal medicine departments during pregnancy. At some point, I came to the hospital six times a month. When appointments for the obstetrics department and the internal medicine department were made on different days, I had to come to the hospital on two days in a row.

<Increased medical bills>

- I usually spend 12,000 - 13,000 yen per month on medical bills. I paid approximately 20,000 yen per month during pregnancy. So, I paid a lot of medical bills.

\section{5) Hope for safe childbirth}

This category comprised the following three subcategories: "surrounding herself with people who show an understanding of pregnancy", "presence of stress until delivery", and "seeing the usefulness of the insulin pump also during pregnancy".

The subcategory "surrounding herself with people who show an understand- 
ing of pregnancy" was composed of the following two codes: <in order to help her husband deepen his understanding of pregnancy, learning together about pregnancy $>$ and <letting a close acquaintance know of her pregnancy $>$; "presence of stress until delivery" had one code, namely <additional stress from pregnancy $>$. The subcategory "seeing the usefulness of the insulin pump also during pregnancy" had one code, namely $<$ feeling the peace of mind when using the familiar insulin pump $>$.

$<$ In order to help her husband deepen his understanding of pregnancy, learning together about pregnancy>

- My husband is very understanding. When I become sick or pregnant, he learns about the condition with me. Rather than learning together, it was like I taught him about my conditions.

<Letting a close acquaintance know of her pregnancy>

- I became pregnant soon after we moved into our current home. So, I was wondering whether I should let neighbors know (of my type 1 diabetes). I ended up letting a close mom friend know of my disease, but not anyone else. If I were self-injecting insulin, people could see me doing so. On the other hand, no one notices that I am using insulin because I am using the insulin pump. So, no one would comment on me even if I am injecting myself (insulin) in the public. No one notices as long as I use the pump quickly.

<Additional stress from pregnancy>

- I feel stress for many different reasons during pregnancy. I do not get overwhelmed by stress.

$<$ Feeling the peace of mind when using the familiar insulin pump >

- I prefer using the insulin pump during pregnancy as well because I have been using it for a long time.

\subsection{Storyline}

Mrs. A's experience of [abnormalities in the delicate body] involved the following pregnancy-related conditions: <development of pain characteristic to injection $>$, <sensitive skin due to gestational itch $>$, and <remaining scars from repeated injections at the same site $>$. Mrs. A experienced physical conditions, "increased sensitivity to pain" and "increased skin vulnerability" that were unknown to her prior to pregnancy. Mrs. A needed to receive higher doses of insulin due to <a surge in blood glucose levels $>$. Meanwhile, she experienced $<$ frequent hypoglycemia due to strict tolerance>, which led to "large fluctuations in blood glucose levels". During pregnancy, "a large increase in insulin doses" was required for her to maintain normal levels of blood glucose. Mrs. A realized that <insulin was not working > during pregnancy, and experienced [difficulty in controlling blood glucose levels for safe childbirth]. Moreover, Mrs. A's doctor instructed her to use "different tools in different stages of pregnancy", i.e., the doctor performed <insulin therapy limited to pregnancy $>$ and changed insulin administration devices $<$ to an insulin pump for safe childbirth $>$. Type 1 diabetes 
does not cause complications in either the mother or the fetus. Therefore, Mrs. A told that she could become pregnant as long as she understood before she became pregnant that "strict blood glucose control would be required during pregnancy", and as long as she satisfied the following conditions: <setting strict HbAlc goals in order to be allowed to become pregnant $>$; <setting strict tolerance during pregnancy $>$ and $<$ requiring hospitalization for blood glucose control>.

Mrs. A experienced "interference with everyday life due to a large belly", such as <choosing the right clothes to hide the insulin pump $>$ and $<$ the large belly was pushing the site where the insulin pump was attached against objects $>$. She told about [inconvenience in everyday life due to a large belly] as she needed to pay greater attention to the insulin pump during pregnancy than she usually did. Moreover, a pregnant woman with type 1 diabetes needs to see both an obstetrician and an internal medicine physician. As Mrs. A needed to visit both departments, and she described those to be "daunting". She mentioned $<$ more frequent doctor visits $>$ and $<$ increased medical bills $>$. These aspects were associated with [the burdens of visiting both obstetrics and internal medicine departments] as they were relevant to Mrs. A during her entire pregnancy.

Mrs A "surrounded herself with people who show an understanding of pregnancy" in building a favorable environment for her pregnancy; for example, <in order to help her husband deepen his understanding of pregnancy, Mrs A learned together with him about pregnancy $>$ and she <let a close acquaintance know of her pregnancy>. Moreover, MrsA experienced the "presence of stress until delivery". Despite the difficulties and restrictions during pregnancy, such as $<$ additional stress from pregnancy $>$ and $<$ feeling the peace of mind when using the familiar insulin pump $>$, she calmly reflected on her experiences by "seeing the usefulness of the insulin pump also during pregnancy". Such experiences are an epitome of her [hope for safe childbirth].

\section{Discussion}

\subsection{Strict Blood Glucose Control for a Pregnant Woman with Type 1 Diabetes}

When a woman with type 1 diabetes becomes pregnant, her blood glucose levels become higher than usual because insulin resistance increases due to the impact of hormones secreted from the placenta. In addition, the body of a fetus is formed by gestational week 8 (two months into pregnancy). A congenital anomaly is likely to occur if the mother's blood glucose levels are high during this period [9], which is likely to cause problems in pregnancy, such as miscarriage, premature birth, polyhydramnios, and fetal macrosomia.

However, in the case of type 1 diabetes, the mother and the baby can be protected from congenital anomaly, miscarriage, and premature birth through means such as planned pregnancy which also covers the pre-pregnancy period and strict blood glucose control [9]. 
Mrs. A's experiences indicated that pregnant women with type 1 diabetes require care for managing both everyday life and the disease, in order to achieve safe childbirth. Takahashi et al. (2016) [10] found that patients with type 1 diabetes can stay positive while they are receiving treatment as long as their physical condition is stable. The categories extracted in this study included terms that suggest negative experiences, such as "abnormality", "difficulty", "inconvenience", and "burden". Mrs. A may have used these negative terms because she strongly felt difficulty in controlling her blood glucose levels.

During the last pregnancy, Mrs. A experienced [abnormalities in her delicate body]. Syringe needles used for insulin injection are thinner than other medical syringe needles. The tip of syringe needles for insulin injection has a special design that reduces pain in the patient; its structure reduces resistance generated when insulin is injected [3]. However, pregnant women who inject themselves with insulin tend to develop gestational itch, gestational eczema, and pigmentation in the early stage of pregnancy during which estrogen levels increase. Moreover, the skin of pregnant women is sensitive to stimulation. Their skin is stretched and becomes vulnerable as their abdomen grows larger. Given this fact, it can be assumed that Mrs. A had skin problems as she injected herself with insulin, which she expressed as part of her experiences of [abnormalities in the delicate body] in which she suffered from pain and itching at the insulin injection sites as well as scars left at the sites.

The doses of insulin that Mrs. A was receiving during pregnancy were much higher than her non-pregnant dosage. The body of a pregnant mother is the source of energy for the growth of the fetus. During pregnancy, there are changes in glucose metabolism in the mother in order to prioritize the transportation of glucose to the fetus. This is a physiological mechanism common among all pregnant women [11]. However, insulin resistance increases in a pregnant woman with type 1 diabetes. This is probably why Mrs. A needed to increase her insulin dosage. Hyperglycemia during pregnancy can be life-threatening to both the mother and her child. It was assumed that the degree of fluctuation in blood glucose levels in Mrs. A increased as her insulin doses were increased. She, therefore, experienced hypoglycemia frequently.

The category [difficulty in blood glucose control for safe childbirth] indicated that it is not easy for a pregnant woman with type 1 diabetes to control their blood glucose levels. A study has found that pregnant women with diabetes feel difficulty in controlling their blood glucose levels [12]. Another study has reported that controlling blood glucose levels within a favorable range can be set as the primary goal in maintaining the health of a diabetic mother during pregnancy [13]. The same was suggested in this study. Mrs. A may have felt difficulty in controlling her blood glucose levels, although she understood that she needed to receive higher doses of insulin during pregnancy in order to control and maintain her blood glucose levels within a favorable range.

Detailed experiences of management methods were extracted from the cate- 
gory [difficulty in preferable blood glucose control for safe childbirth]. The experiences were as follows: satisfying the conditions for obtaining permission to become pregnant; planned pregnancy; setting the tolerance of HbAlc, hospitalization for blood glucose control, and insulin therapy limited to pregnancy. A study has reported that pregnant women with diabetes can be motivated to control their blood glucose levels when they are hoping to give birth to a healthy baby [13]. This finding may also apply to Mrs. A. Meanwhile, it can also be assumed that she could control her blood glucose levels partly because she needed to do so only during pregnancy. Moreover, Mrs. A talked about the usefulness of insulin pump therapy, although she did not mention her resistance against inserting a needle into the abdomen. Mrs. A had an onset of type 1 diabetes before she became pregnant. The narrative of Mrs. A suggested that administering insulin and using administration devices had already been part of her everyday life.

The category [inconvenience in everyday life due to a large belly] indicated that Mrs. A experienced the growth of her abdomen as her pregnancy progressed. As her abdomen became larger and rounder, the site, where the insulin pump was attached, was pushed forward. Hence, Mrs. A chose clothes that can hide the insulin pump. She was feeling inconvenience with the site where the insulin pump was attached pushing against an object. Takii et al. (2013) [14] pointed out that patients with type 1 diabetes suffer from misunderstanding and prejudice from others who do not understand the disease. Findings from this study also suggested that Mrs. A was using various methods and behaviors in order to hide the insulin pump because she was frustrated when people around her paid excessive attention to her disease. It was necessary for Mrs. A to protect her belly where the insulin pump was attached. Her narrative suggested that she needed to think about how to avoid any obstacles that she would face as a result of using the insulin pump. A study on the usefulness of an insulin pump in everyday life [3] found that the presence of the insulin pump and limitations in movement when the pump is worn can cause users to feel inconvenience in everyday life. Given this, it can be said that pregnant women who are using an insulin pump may feel the presence of the pump and limitations in movement more strongly compared to those who are not pregnant. This is new knowledge generated from this study.

For the category [the burden of visiting both obstetrics and internal medicine departments], Mrs. A pointed out the increased frequency of hospital visits because she needed to see doctors in both departments. Such a situation is not limited to Mr.s A. Any woman with type 1 diabetes needs to see both an obstetrician and an internal medicine physician during pregnancy. According to Kato et al. (2013) [12], women with type 1 diabetes feel that health professionals in obstetrics tend to lack the real-world knowledge and experience of the disease. In their study, participant women stated that they are afraid of fully trusting such health professionals. Mr.s A's thoughts in this aspect were not clear because she 
did not mention any negative experiences in relation to health professionals in obstetrics.

When it comes to the category [hope for safe childbirth], not only Mrs. A but all mothers-to-be are hoping to give birth to a healthy baby. According to Tanaka et al. (2012) [15], the frequency of hypoglycemia and fluctuation in blood glucose levels significantly changes in women with diabetes when they become pregnant. The researchers reported that it is therefore important to build an environment that helps mothers with diabetes seek support from others at any time so that they can safely give birth to their baby. It was assumed that, in order to safely give birth to her baby, Mrs. A prepared an environment where her husband and close friends could understand her condition. Additionally, according to Sakai et al. (2003) [16], patients with type 1 diabetes are hoping that they can achieve milestones in life, such as employment, marriage and childbirth, and child-rearing. The same findings were obtained from this study: Mrs. A mentioned her wish to give birth to a healthy baby in her narrative probably because such an experience is a milestone in life.

\subsection{Implications for Clinical Practice}

Experiences during the pregnancy of a patient with type 1 diabetes suggest that women can give birth to a healthy baby even if they have the disease. This suggestion carries important implications. Of particular note is that Mrs. A became pregnant after her blood glucose levels improved. She achieved this outcome by consulting physicians when she was wishing to become pregnant. Also, it is important in patients with type 1 diabetes that the patients are required to prioritize strict blood glucose control in every aspect of life during pregnancy. If the blood glucose levels of the mother are poorly controlled, both the lives of the mother and fetus will be at risk. Additionally, persistent hyperglycemia may lead to miscarriage, premature birth, polyhydramnios, congenital anomaly, and fetal macrosomia. Repeated hypoglycemia poses a physical burden to the patient. On the other hand, a patient with well-controlled blood glucose can maintain stable physical and psychological conditions during pregnancy, creating a positive cycle that helps the patient lead her everyday life. Mrs. A and her fetus were in a good condition throughout pregnancy, and Mrs. A safely delivered the baby. She achieved this outcome probably because she rigorously controlled her blood glucose levels prior to childbirth.

Mrs. A had a strong wish to give birth to a healthy baby; therefore, she became pregnant in a planned manner under the supervision of her doctor. Furthermore, findings from this study indicated that Mrs. A was convinced that strict blood glucose control was necessary. It is important to maintain close communication with a pregnant patient with type 1 diabetes in order to understand the state of blood glucose control in the patient as well as her thoughts about pregnancy and delivery.

Women with type 1 diabetes who are pregnant and giving birth are at a higher 
risk of death compared to healthy women. Thus, it is natural that pregnant women with the disease are extremely anxious until they give birth to their babies. It is important for a nurse to work together with a pregnant patient with type 1 diabetes in setting goals, and to provide the patient with support for her daily activities. In order to achieve these, the nurse needs to ask the patient to talk about her everyday life. In doing so, the nurse may need to take into account the patient's values and provide her with information, knowledge, skills, and psychological support as necessary. Moreover, in order to help such a patient safely give birth to her baby, it is important to encourage the patient to have her husband understand the key aspects of pregnancy and childbirth, as well as seeking his support. In such cases where a woman with type 1 diabetes wishes to become pregnant, the following practices are key: frequently providing the patient with in-depth information; working together with the patient in setting goals; teaching the patient how to control blood glucose levels, and providing the patient with support in every aspect of everyday life through the entire course of care.

\section{Conclusion}

This study extracted the following five experiences from a narrative of pregnancy of a patient with type 1 diabetes: [abnormalities in the delicate body]; [difficulty in blood glucose control for safe childbirth]; [inconvenience in everyday life due to a large belly]; [the burden of visiting both obstetrics and internal medicine departments]; and [hope for safe childbirth]. Mrs. A gave her account on strict blood glucose control and difficulties that she faced in everyday life. However, Mrs. A had a wish to give birth to a healthy baby; therefore, she became pregnant in a planned manner under the supervision of her doctor. Additionally, findings from this study indicated that Mrs. A strictly controlled her blood glucose levels knowing its importance. In cases where a woman with type 1 diabetes wishes to become pregnant, it is important for a nurse to practice the following: frequently providing the patient with in-depth information; working together with the patient in setting goals, and providing the patient with appropriate support in controlling blood glucose levels.

\section{Limitations}

This study was a case study. It is assumed that the categories and subcategories of this study reflect the individual situation of the participant patient. Hence, findings from this study are not necessarily applicable to all women with type 1 diabetes. Future studies need to examine a larger number of cases; study methods need to be refined so that more experiences of pregnancy in women with type 1 diabetes can be extracted.

\section{Acknowledgements}

The authors would like to express sincere gratitude to the study participant for 
her willingness to participate in the interview as well as the people who introduced her to them.

\section{Conflicts of Interest}

The authors declare no conflicts of interest regarding the publication of this paper.

\section{References}

[1] Diabetes Clinical Data Management Study Group (2019). http://jddm.jp/

[2] Uchigata, Y. (2015) To Become Mother Whether She Has Diabetes or Not. Diabetes \& Pregnancy, 15, 8.

[3] Nishio, I. and Chujo, M. (2017) Comparing Lifestyle Convenience of Multiple Daily Injection and Continuous Subcutaneous Insulin Infusion. Journal of Japan Academy of Diabetes Education and Nursing, 21, 63-68.

[4] Nishio, I. and Chujo, M. (2014) The Suffering from Illness Experience of Young Adult with Type 1 Diabetes. The Journal of the Yonago Medical Associatio, 65, 49-56.

[5] Nishio, I. and Chujo, M. (2016) Qualitative Analysis of the Resilience of Adult Japanese Patients with Type 1 Diabetes. Yonago Acta Medica, 59, 196-203.

[6] Nishio, I., Chujo, M. and Kataoka, H. (2016) A Qualitative Study of Confusing Experiences among Japanese Adult Patients with Type 1 Diabetes. Yonago Acta Medica, 1, 81-88.

[7] Motoshima, Y., Ohmura, C., Shimizu, T., et al. (2009) The Actual Report of Pregnancy Diabetes Mellitus and Pregnancy in Diabetic in Our Department. Practice, 26, 209-215.

[8] Omori, Y. (1992) Latist Issues in Diabetes Mellitus and Pregnancy. The Medical Frontline, 55, 88-92.

[9] The Japan Diabetic Association (2019) Japanese Clinical Practice Guideline for Diabetes 2019. 284-297.

[10] Takahashi, Y., Tanimoto, M. and Masaki, H. (2013) The Role of Interpersonal Nursing Skills in Promoting Self-Care among Diabetic Patients in an Outpatient Setting. Journal of Japan Academy of Diabetes Education and Nursing, 17, 113-123.

[11] Tanaka, K. (2018) Support Network for Women's Health for Diabetic Women. Journal of Japan Academy of Diabetes Education and Nursing, 22, 66-71.

[12] Kato, Y., Tanaka, K. and Nakashiima, K. (2013) The Psychological Experiences of Diabetic Women during Pregnancy and Delivery. Maternal Health, 53, 458-466.

[13] Abe, H., Takahsashi, A., Saito, M., et al. (2009) Thoughts (or Feelings) about Pregnancy, Delivery and Postpartum Period Held by Women Who Were Diagnosed with Irregular Sugar Metabolism after Becoming Pregnant. Journal of the Japanese Red Cross Society of Nursing Science, 9, 1-9.

[14] Takii, M. (2013) Psychosomatic Aspects of Type 1 Diabetes. Japanese Journal of Psychosomatic Medicine, 53, 12-19.

[15] Tanaka, K., Oda, K., Kawamura, T., et al. (2012) Self-Care Behavior to Maintain Good Glycemic Control during Pregnancy and Child Rearing Based on the Experiences of Women with Diabetes Mellitus. Osaka Medical College Journal of Nursing Research, 2, 21-28. 
[16] Sakai, M., Sawada, A. and Hirose, Y. (2003) Components of Hope in Diabetic Patients of Adolescent-Onset Type 1 and Nursing Supports. The Journal of the Nursing Society of the Toyama Medical and Pharmaceutical University, 5, 49-59. 